\title{
Glycosylated Recombinant Human Interleukin-7
}

National Cancer Institute

\section{Source}

National Cancer Institute. Glycosylated Recombinant Human Interleukin-7. NCI

Thesaurus. Code C77879.

A glycosylated recombinant protein which is chemically identical to or similar to endogenous human interleukin-7 (IL-7) with hematopoietic and immunopotentiating activities. Naturally produced by bone marrow, thymic stromal, and spleen cells, the cytokine interleukin-7 is a hematopoietic growth factor for progenitor B cells and T cells, stimulating proliferation and differentiation of mature T-cells and natural killer (NK) cells. As with glycosylated endogenous proteins, glycosylation of recombinant proteins may fundamentally affect their biological activity, function, clearance from circulation, and antigenicity; glycosylation of recombinant proteins that are chemically idenitical to or similar to endogenous proteins may render protein structures and biological activities that are more similar to those of glycosylated endogenous proteins. 